\title{
On the Way to
}
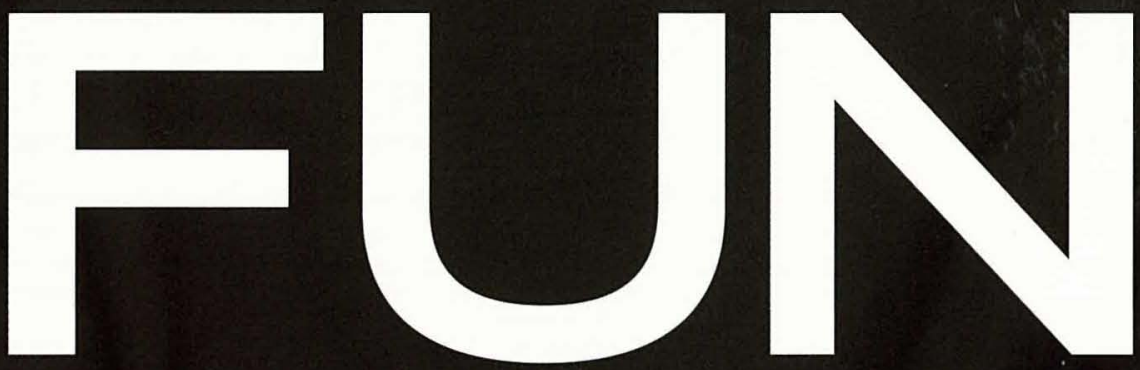

An Emotion-Based Approach to Successful Game Design

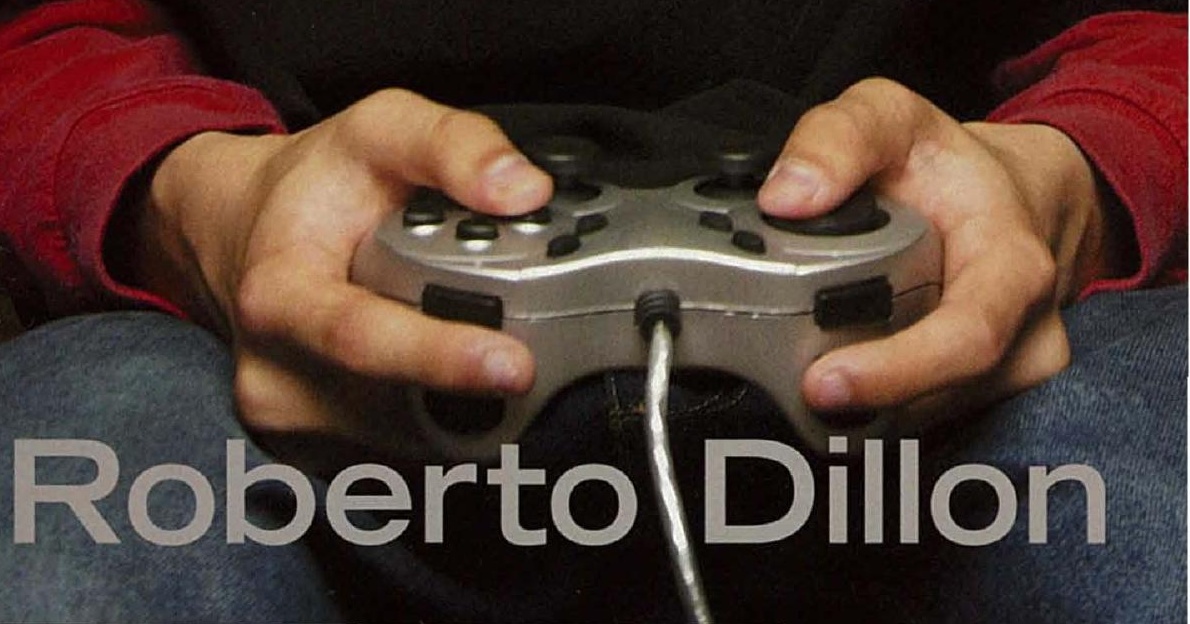


On the Way to Fun 



\section{On the Way to Fun}

An Emotion-Based Approach to

Successful Game Design

Roberto Dillon

DigiPen Institute of Technology

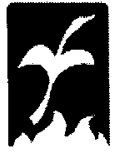

A K Peters, Ltd.

Natick, Massachusetts 
Editorial, Sales, and Customer Service Office

A K Peters, Ltd.

5 Commonwealth Road

Natick, MA 01760

www.akpeters.com

Copyright $@ 2010$ by A K Peters, Ltd.

All rights reserved. No part of the material protected by this copyright notice may be reproduced or utilized in any form, electronic or mechanical, including photocopying, recording, or by any information storage and retrieval system, without written permission from the copyright owner.

All logos, trademarks, games, and systems are properties of their respective owners.

\section{Library of Congress Cataloging-in-Publication Data}

Dillon, Roberto.

On the way to fun : an emotion-based approach to successful game design / Roberto Dillon.

p. $\mathrm{cm}$.

Includes bibliographical references and index.

ISBN 978-1-56881-582-4 (alk. paper)

1. Computer games-Programming. 2. Computer games-Design-

Social aspects. I. Title.

QA76.76.C672D54 2919

$794.8^{\prime} 1526-\mathrm{dc} 22$

2009050148

Cover image $(\mathcal{C}$ Veer Incorporated.

Printed in the United States of America 1413121110

10987654321

JAWESOOOK

UNIVERSITY

LIBRARY 
To Jing 



\title{
Table of Contents
}

\author{
xi Foreword \\ xiii Preface \\ xv Introduction: What Makes a Game Fun?
}

1 Emotions and Games: The 6-11 Framework

3 What Are Basic Emotions and Instincts?

7 Introducing the 6-11 Framework

17 Contextualizing the 6-11 Framework

23 Emotional Analysis of a Gameplay Session

27 Making Fun Games the Emotional Way

\section{Case Studies: Retro Games}

35 Why Retro Games?

36 - Computer Space Vs. Space Invaders

$40 \cdot$ Asteroids

44. Battlezone 


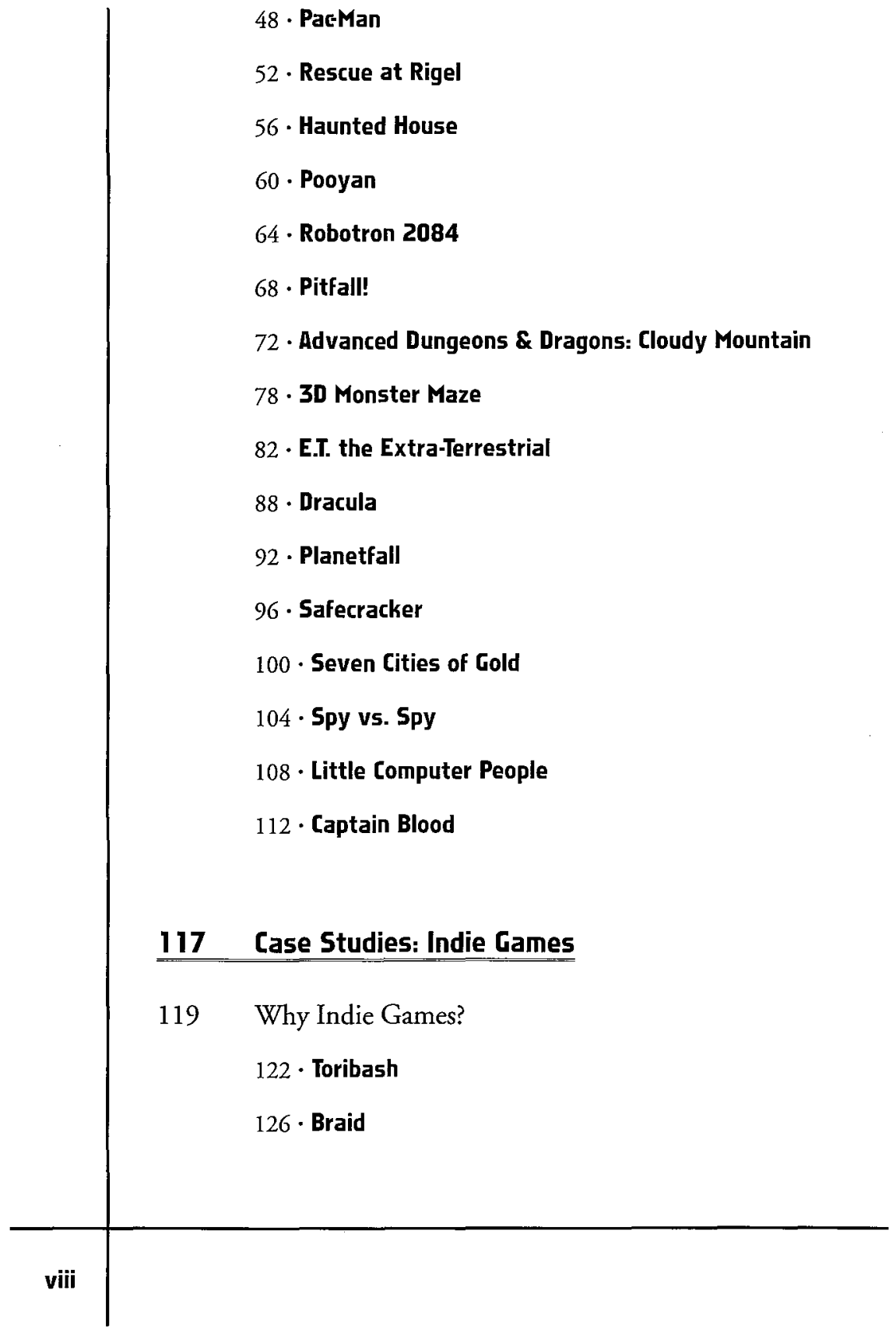


130 - I Wish I Were the Moon

134 - Castle Crashers

$138 \cdot \operatorname{Tag}$

142 . Flower

146 - Plants vs. Zombies

150 World of Goo \& Crayon Physics Deluxe

\section{Conclusions}

157 In the End

165 References

167 Index 



\section{Foreword}

Have you ever felt really happy and joyful or really angry because of playing a video game? How often do you experience those deeply emotional moments in games? I remember quite vividly when I angrily punched my Game Boy's screen while playing Double Dragon, cracking the screen, and how happy I was when I finally beat the boss that gave me so much trouble. I remember my amazement during the opening of Half-Life and when I couldn't stop laughing as $\mathbb{b}$ clicked my way through Monkey Island. And the several occasions of breaking into tears playing Shadow of the Colossus are following me around to this day. But despite all the games I have played, there are less than a handful that I still remember vividly, if at all.

When I started to get interested in game design and able to analyze games properly, I quickly realized that what most differentiates games from any other medium is not their interactivity, but their lack of emotional impact. The fact that you would really care about a game character or the game world was, for most games, not a priority.

When I had the opportunity at the beginning of my career to join Lionhead Studios to work on Black \& White and learn about game design from Peter Molyneux, it became quite clear why he was hailed one of the geniuses of the industry. Almost a year after my short stint in the UK, I fortuitously met Shigeru Miyamoto and was able to probe his brain for a bit. I learned that he has a very similar thought about games-the player needs to get attached to something in the game world and really care about it. Then the player experiences the game hand-inhand with this object of attachment and thus the game creates emotional moments. This notion is not limited to story-driven 
games. Any game can and should create those moments. Some are very easy to create, but the ones with most impact are tough. I made it a rule from then on that everything I design must consider the emotional reaction of the user. That premise should be applied to any kind of design, not just games.

During the past year, I worked closely with Roberto on the Music and Emotion Driven Game Engine (M-EDGE) research project, and finally had the opportunity to incorporate emotion into games in a way almost unheard of before. Roberto helped me to design a game that could, like a living being, have a real emotional reaction to music, and have an impact on the game world as well as the player. It sure did change our moods very quickly at times.

Roberto's expertise in music and his experience with emotional systems became very quickly a valuable resource for me. $\mathrm{He}$ is someone who, with his dedication, critical thinking, and decisiveness brought out the best in our endeavor of creating a game with emotional consideration and impact. Both of us have had the good fortune to experience the world in many ways and his openness to new ideas helped me progress in my own field.

Roberto is an excellent researcher who is looking at the advancement of game development thîrough new and sometimes unusual means, and who doesn't take things for granted. I wish many of the points raised here by Roberto had been as explicitly written out much earlier-maybe I wouldn't have broken my Game Boy in the first place. I hope this book will become a very valuable tool for you too. 


\section{Preface}

I spent the last few weeks commuting to and from work on the bus while carefully studying The Art of Computer Game Design by Chris Crawford [Crawford 84]. Needless to say, I enjoyed that pioneering book tremendously, not only for the actual contents, which are still mostly valid and able to make us really think about game design, but also for Crawford's concision and synthesis skills. In just about 80 pages, he could clearly explain his ideas. Crawford's book was the first one dedicated to videogame design. Countless more followed and today game design books are getting extremely comprehensive, insightful, and...massive.

This is obviously very exciting for all of us who have an interest in games, whether as a profession or as a hobby. But for those who, like me, keep trying to squeeze reading and studying into our busy daily work schedules, reading a $1000+$ page tome on a crowded bus or subway train isn't an easy task at all! This was one of my concerns when I started writing this little book. I wanted to be as concise and focused as possible and deliver my message in a small, agile book that could easily be carried around and read anywhere whenever there are five minutes free.

With respect to the actual contents, my objective was to provide some food for thought by clarifying which emotions and instincts are particularly relevant within the gaming medium and how they can interact with each other to enhance the gaming experience. This can hopefully help us with the oldest design problem of all — how to make a "fun" game- by giving us a fresh perspective on how to properly design and develop gameplay ideas in a way that makes these interactions straightforward and natural. 
In doing this, I wanted to recall some old and fond memories in those who, like me, are old enough to have witnessed the beginning of our industry. I also hoped to excite some curiosity among the new generations by analyzing several case studies coming from the so-called "retro gaming" scene, in particular by focusing on those games that were published between the late seventies and mid-eighties.

Overall, this book was written for aspiring and beginning game designers who want to know a little more about human nature, emotions, and instincts and their important place in designing games. I am also confident that, by formalizing concepts in a simple and straightforward way that were usually left to academic studies, the book may even be of some help to more experienced professionals by making them aware of something they may well have already used, but only at a subconscious level thanks to their own personal intuition and skills.

Roberto Dillon

Singapore

July 4, 2009 


\title{
Introduction: What Makes a Game Fun?
}

\author{
Art is something designed to evoke emotion through \\ fantasy... The compurer game is an art form because \\ it presents its audience with fantasy experiences that \\ stimulate emotion.

$$
\begin{array}{r}
\text {-Chris Crawford } \\
\text { The Art of Computer Game Design }
\end{array}
$$

What makes a game fun? And what is "fun" actually? Unfortunately providing an exhaustive and "objective answer to these legitimate questions is likely to be an impossible task since having fun is a very personal activity that can be completely different from individual to individual.

In fact, what is fun for someone can be extremely boring for somebody else and vice versa. For example, some people find watching a football match to be an extremely fun and exhilarating activity, while others would definitely skip it and spend their free time doing something else. Videogames are no exception, and even within them there are many subcategories that attract and provide fun experiences to only a specific group of people. So the aim of this small book is not to try to directly answer "what makes a game fun" but rather to provide a simple and easy to understand framework that links successful yet simple games to basic human emotions and instincts.

Why? Well, the idea is to show aspiring and beginning game designers how certain games successfully relied on behavioral aspects deeply rooted in human beings to provide experiences that completely immersed players in those experiences. In doing this, we are assuming that such games were able to deliver a truly 
entertaining experience and we will analyze the reasons why the games were so engaging that they were ultimately perceived as fun-however we define this concept.

The book is structured in different parts. The first part provides a theoretical framework for analyzing gameplay in terms of underlying emotions. The proposed model is called the "6-11 Framework" after the 6 basic emotions and 11 instincts it takes into consideration. In particular, after a quick introduction to basic emotions and instincts, we discuss which ones are more relevant in videogames and how they can be used to affect and influence players' experiences. To do this, we will define a "path", or "way", that, by having different emotions and instincts as intermediate stops, will likely lead us to the final destination of "fun".

The remaining parts are dedicated to a collection of case studies with a special focus on old retro games. In Part II, we will review and analyze a selection of 20 meaningful examples from the 1970s and 1980s to show how, by basing their gameplay on a few of the emotions and instincts considered in the 6-11 Framework, they succeeded in engaging generations of players despite being developed with very limited technical means and without any fancy graphics or realistic sounds. Alongside these, we discuss a couple of games that didn't live up to their expectations. This will be instructive and also a little provocative as we will try to explain the reasons for their partial or complete failure by pointing out how they overlooked or misinterpreted some particular emotion or instinct which resulted in the players feeling less engaged and immersed in the overall gaming experience. In Part III we will look at how the proposed framework could be applied to more contemporary productions by analyzing a few well-known modern independent ("indie") games. Finally, Part IV will conclude with some thoughts about the broader picture of the usefulness of our approach. 